\title{
The Importance of Ventral Premotor Cortex for Body Ownership Processing
}

\author{
Robin Bekrater-Bodmann, Jens Foell, and Sandra Kamping \\ Department of Cognitive and Clinical Neuroscience, Central Institute of Mental Health, 68159 Mannheim, Germany \\ Review of Zeller et al.
}

The perception of one's own hand is an eminently coherent impression: proprioceptive, tactile, and visual inputs usually correspond perfectly. Current theoretical frameworks postulate that this multimodal integration is required for a feeling of ownership of the body, and is ultimately accompanied by a sense of self. This might seem trivial; but actually, it is necessary for the discrimination between one's own body and its surroundings, as well as the distinction between self and other. However, how the brain manages the feeling of body integrity with all its parts remains an open question.

The major mystery of body perception can be expressed by this simple question: Why do I perceive my hand as belonging to me? The ventral premotor cortex $(\mathrm{PMv})$ seems to play a key role in this integrative process. Early studies showed that multimodal receptive fields in the $\mathrm{PMv}$ of primates appear to combine visual and somatosensory input to locate one's own limb in peripersonal space (Graziano et al., 2000). Botvinick and Cohen (1998) complemented these findings by showing that a visible rubber hand is experienced as belonging to the subject's body when

\footnotetext{
Received May 9, 2011; revised May 26, 2011; accepted May 26, 2011.

This work was supported by the PHANTOMMIND advanced grant of the European Research Council (FP7/2007-2013)/ERC Grant Agreement No. 230249.

Correspondence should be addressed to Robin Bekrater-Bodmann, Department of Cognitive and Clinical Neuroscience, Central Institute of Mental Health, Square J 5, 68159 Mannheim, Germany. E-mail: r.bekrater-bodmann@ zi-mannheim.de.

DOI:10.1523/JNEUROSCI.2302-11.2011

Copyright $\odot 2011$ the authors $\quad 0270-6474 / 11 / 319443-02 \$ 15.00 / 0$
}

both the rubber hand and the subject's own hand are stimulated congruently in terms of location and temporal consistency. The authors assumed an involvement of the PMv in this so-called rubber hand illusion (RHI), which was confirmed by Ehrsson et al. (2004) using functional magnetic resonance imaging, indicating that activity in PMv is positively correlated with the experience of body ownership. However, it remained unclear whether multisensory integration within the PMv is necessary to generate a feeling of body ownership, or whether the neuronal processes in the RHI paradigm are unique and different from usual body perception processes.

This question was recently examined by Zeller and colleagues (2011). The authors assessed the experience of illusory ownership of an artificial limb in patients suffering from circumscribed unilateral ischemic strokes, which damaged the basal ganglia and periventricular white matter, affecting the putamen, precentral and postcentral cortices, and the thalamus. To evaluate the importance of lesion side, the RHI was induced both for the ipsilesional and contralesional side of the body, and was rated by using an RHI perception questionnaire. In doing so, the authors assessed the prevalence of RHI failure (RHIF), which was defined as a lack of the sensation of perceived ownership of the artificial limb. Additionally, most of the participants were asked how strongly they experienced the contralesional hand as belonging to themselves, assessing the presence of asomatognosia following stroke. These subjective responses were related to the degree of lesion by performing voxel-based lesion-symptom mapping (VLSM) to identify affected voxels that significantly correlated with the perception scores. These voxels were used as seed areas for a subsequent investigation using probabilistic diffusion tractography (PDT) in an averaged healthy brain to identify subcortical white matter tracts passing through these regions.

The authors observed contralesional RHIF in $25.7 \%$ and additional ipsilesional RHIF in $10.0 \%$ of the stroke patients. However, RHIF was not necessarily associated with asomatognosia, which was found in $28.1 \%$ of the patients. The inability to perceive illusory body ownership during RHI induction was associated with clearly defined white matter lesions, whereas control responses did not correlate with brain damage, indicating a specific relation between the experience of illusory body ownership and connecting fibers. PDT for the seed regions obtained in the VLSM showed that both RHIF and asomatognosia were associated with connections between frontal and parietal cortices, frontal operculum, thalamus, basal ganglia, and the cerebellum. The only prominent difference was an additional strong connection to PMv for the RHIF analysis, which was lacking in the asomatognosia analysis. The authors interpreted these results as evidence for causal neuronal involvement of the PMv in illusory body ownership experience. 
Zeller and colleagues' (2011) results have implications for the study of body perception in general. Their findings indicate that the inability to perceive illusory ownership of an artificial limb (i.e., RHIF) and the loss of ability to recognize that a body part belongs to one's own body (i.e., asomatognosia) might involve two different neuronal processes in at least partially different brain areas. The consequence of this finding, namely that body ownership itself is not necessarily mediated by the $\mathrm{PMv}$, is an important merit of the study, complementing convincingly the theoretical concept of the function of PMv.

Gentile et al. (2011) showed that the $\mathrm{PMv}$ reacts in an additive manner to incoming sensory input; i.e., the PMv is more active during visuotactile stimulation of one's own hand than during visual perception or tactile stimulation alone, indicating an integrative, stimulationlinking role of the PMv, which was also shown for the posterior parietal cortex (PCp) (Nakashita et al., 2008). Activity in both structures is positively correlated with vividness of RHI perceptions (Ehrsson et al., 2004), complementing findings which showed that both the PCp and the PMv can bridge the gap between multimodal sensory mismatches (Bremmer et al., 2001). The results reported in the present study suggest that stroke patients affected by lesions in the white tissue connecting the PMv with other brain regions were less able to integrate proprioceptive, visual, and tactile stimuli into a corresponding event, which might be the basis for the experience of RHI sensations, but not necessarily for common body ownership.

We would, however, like to point out that there is reason to suspect that the prevalence of RHIF due to stroke given in the study by Zeller and colleagues (2011) may be based on a faulty assumption: previous studies (e.g., Ehrsson et al., 2004) found that $\sim 25 \%$ of healthy participants are RHI non-responders; i.e., they are inherently unable to perceive the illusory perceptions induced by the RHI paradigm, even in the absence of structural failure. The RHIF rate of stroke patients reported in the present study is similar to this baseline. Further, the RHIF prevalence found in an additional healthy control sample might be underestimated (7.5\% unilateral and $2.5 \%$ bilateral RHIF), suggesting that behavioral approaches to quantify illusory body ownership (Botvinick and Cohen, 1998) are necessary to evaluate the present results.

One additional peculiarity of the study by Zeller and colleagues (2011) is the lack of consistent cortical lesions associated with asomatognosia, especially within the parietal cortex (Feinberg et al., 2010), suggesting that the present method to assess this complex perceptional feature by questionnaire might not be sufficient, due to the abstract nature of this phenomenon. As in the assessment of RHIF, behavior-based approaches (Feinberg et al., 2010) might be more appropriate to obtain a valid diagnosis of asomatognosia.

Nevertheless, in light of the results by Zeller and colleagues (2011), the PMv has to be regarded as a gate that must be passed through to link multimodal sensory input and to incorporate external objects into the body representation, but not to create ownership itself. What could be the natural use of this function, when no artificial hands have to be incorporated into the body? Presumably, it is essential for the ability of primates to use tools. PMv, PCp, and other cortical areas are activated during tool use (Macaluso and Maravita, 2010), indicating that a widely distributed network involving frontal and parietal areas is geared to interact with the environment as immediately as possible. The PMv is a candidate region to act as a mediator between the internal and the external representation of the environment by several functions, which can be summarized as follows: (1) coding for the peripersonal space as well as its extensions, (2) coding for postural features of body parts within the peripersonal space, (3) ability to integrate multimodal sensory input, and (4) close connections to other body perception areas (such as the PCp and the insular cortex). As supported by the data from Zeller and colleagues (2011), the sensations during the RHI paradigm might be an involuntary consequence of these functions. This simple experimental setup may therefore be considered an important window into the mechanisms that allow us to pick up an object and to use it as an extension of ourselves.

In conclusion, Zeller and colleagues (2011) have taken an important and necessary step in the study of which paths the brain uses to generate the experience of body ownership. Even taking into account our critical points, they illuminate the importance of PMv for a special kind of body ownership processing and therefore filled a critical gap in the research of body perception.

\section{References}

Botvinick M, Cohen J (1998) Rubber hands 'feel' touch that eyes see. Nature 391:756.

Bremmer F, Schlack A, Shah NJ, Zafiris O, Kubischik M, Hoffmann K, Zilles K, Fink GR (2001) Polymodal motion processing in posterior parietal and premotor cortex: a human fMRI study strongly implies equivalencies between humans and monkeys. Neuron 29:287-296.

Ehrsson HH, Spence C, Passingham RE (2004) That's my hand! Activity in premotor cortex reflects feeling of ownership of a limb. Science 305:875-877.

Feinberg TE, Venneri A, Simone AM, Fan Y, Northoff G (2010) The neuroanatomy of asomatognosia and somatoparaphrenia. J Neurol Neurosurg Psychiatry 81:276-281.

Gentile G, Petkova VI, Ehrsson HH (2011) Integration of visual and tactile signals from the hand in the human brain: an FMRI study. J Neurophysiol 105:910-922.

Graziano MS, Cooke DF, Taylor CS (2000) Coding the location of the arm by sight. Science 290:1782-1786

Macaluso E, Maravita A (2010) The representation of space near the body through touch and vision. Neuropsychologia 48:782-795.

Nakashita S, Saito DN, Kochiyama T, Honda M, Tanabe HC, Sadato N (2008) Tactile-visual integration in the posterior parietal cortex: a functional magnetic resonance imaging study. Brain Res Bull 75:513-525.

Zeller D, Gross C, Bartsch A, Johansen-Berg H, Classen J (2011) Ventral premotor cortex may be required for dynamic changes in the feeling of limb ownership: a lesion study. J Neurosci 31:4852-4857. 